03

\title{
Методика определения пространственного распределения концентрации газа в сверхзвуковых струях при истечении \\ в вакуум
}

\author{
(ㄱ М.В. Петренко, С.В. Бобашев, Г.К. Тумакаев \\ Физико-технический институт им. А.Ф. Иоффре РАН, Санкт-Петербург \\ E-mail: m.petrenko@mail.ioffe.ru
}

Поступило в Редакцию 17 июля 2016 г.

\begin{abstract}
Предложена экспериментальная методика определения пространственного распределения концентрации газа в сверхзвуковых струях миллиметрового размера при истечении в вакуум, основанная на визуализации струи путем ее подсветки лазерно-плазменным источником излучения и обработке полученного распределения интенсивности собственного свечения струи. Измерено пространственное распределение интенсивности свечения струи при ее визуализации. Определено распределение концентрации газа в струе и проведено сравнение результатов эксперимента с расчетными данными, полученными методом численного гидродинамического моделирования.
\end{abstract}

DOI: 10.21883/PJTF.2017.09.44574.16441

В настоящее время компактные сверхзвуковые газовые струи миллиметрового размера рассматриваются в качестве мишеней для формирования лазерно-плазменных источников коротковолнового излучения, применимых в литографическом процессе [1-4] и микроскопии [5]. Задача повышения выхода коротковолнового излучения из плазмы в таких источниках предполагает среди прочего оптимизацию газовой струи [6], что требует разработки методов контроля ее плотности за время истечения. Целью данной работы является определение пространственного распределения концентрации газа внутри струи, что позволит экспериментально оценивать степень ее оптимизации для использования в лазерно-плазменном источнике излучения.

Предлагаемая в данной работе экспериментальная методика определения распределения концентрации газа в сверхзвуковой струе, 


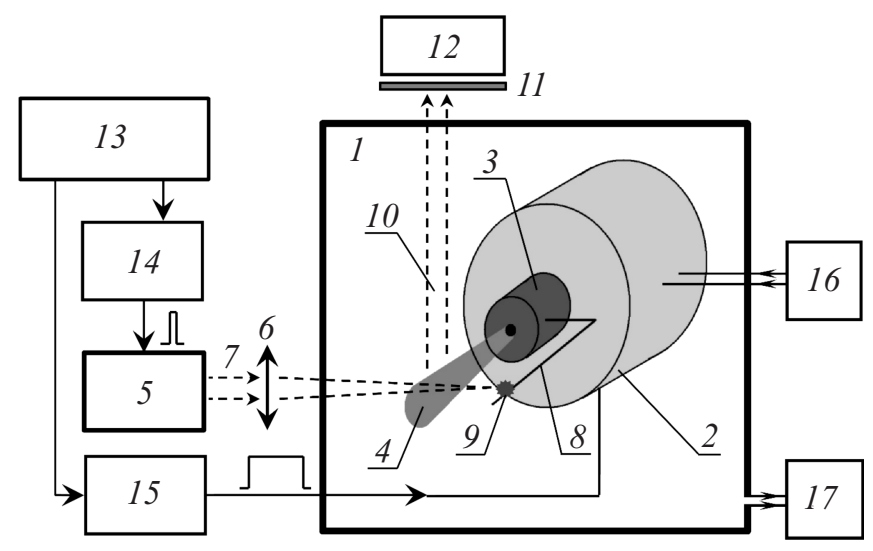

Рис. 1. Схема экспериментальной установки. Обозначения см. в тексте статьи, 13 - система синхронизации, 16 - система подачи газа, 17 - система откачки.

истекающей в вакуум, включает в себя два этапа. На первом этапе осуществляется визуализация струи путем ее подсветки точечным лазерно-плазменным источником излучения. Данная методика может представлять отдельный интерес в качестве средства визуального контроля течения струи. На втором этапе, используя полученное пространственное распределение интенсивности собственного свечения струи, определяется распределение концентрации газа.

Эксперименты по визуализации сверхзвуковой газовой струи миллиметрового размера, истекающей в вакуум, проводились на установке, схема которой представлена на рис. 1. В вакуумную камеру 1 размером $22 \times 12 \times 12 \mathrm{~cm}^{3}$, откачиваемую до давления $10^{-2}$ Torr, помещена система формирования импульсной сверхзвуковой газовой струи, состоящей из камеры высокого давления с электромагнитным клапаном 2 и сверхзвукового сопла Лаваля 3 , обладающего следующими характеристиками: сопло коническое, радиус входного отверстия $r_{i n}=0.1 \mathrm{~mm}$, радиус выходного отверстия $r_{e x}=0.6 \mathrm{~mm}$, длина сопла $l_{n o z}=15 \mathrm{~mm}$, угол раствора $\theta=4^{\circ}$. Электромагнитный клапан работает при значениях входного давления до $10 \mathrm{~atm}$ и позволяет создавать импульсную струю 4 со временем истечения 20-30 ms. В качестве рабочего газа в экспериментах используется ксенон.

Письма в ЖТФ, 2017, том 43, вып. 9 
Твердотельный импульсный лазер на неодимовом стекле 5 , состоящий из генератора и двух усилителей, создает импульс лазерного излучения с длиной волны $1.06 \mu \mathrm{m}$, длительностью импульса $40 \mathrm{~ns}$ и энергией излучения в импульсе до $600 \mathrm{~mJ}$. Работа блоков питания лазера 14 и электромагнитного клапана 15 синхронизована таким образом, что лазерный импульс следует через $15 \mathrm{~ms}$ после начала импульса открытия клапана. Лазерное излучение 7 фокусируется линзой $6(f=111 \mathrm{~mm})$ на молибденовую мишень 8 , находящуюся вблизи газовой струи, в пятно размером $\sim 0.1 \mathrm{~mm} \mathrm{c}$ плотностью потока лазерного излучения в точке фокуса $1.5 \cdot 10^{11} \mathrm{~W} \cdot \mathrm{cm}^{-2}$. При взаимодействии лазерного излучения с мишенью образуется короткоживущая лазерная плазма 9 с температурой $\sim 30 \mathrm{eV}$ и временем жизни $\sim 10 \mathrm{~ns}$, излучающая в широком спектральном диапазоне вплоть до мягкого рентгеновского излучения [7]. Излучение лазерной плазмы возбуждает атомы газовой струи. Рекомбинационное излучение ксенона, отвечающее оптической части спектра 10 , выделяется системой оптических фильтров 11 и регистрируется коммерческим фотоаппаратом Minolta DImage Z1 12.

На рис. 2 показаны результаты эксперимента по визуализации сверхзвуковой струи при давлении на входе сопла $8 \mathrm{~atm}$, энергии лазерного импульса $450 \mathrm{~mJ}$ и расстоянии от молибденовой мишени до оси струи $7.5 \mathrm{~mm}$. На рис. 2 представлена фотография собственного свечения струи, полученная с использованием двух оптических фильтров НC-2 с выдержкой фотоаппарата $1 \mathrm{~s}$. Также на рис. 2 приведены пространственный масштаб и выбранные для дальнейшего исследования оси координат: $z$ - координата вдоль оси струи с началом в точке $O$, соответствующей срезу сопла; $r-$ координата, перпендикулярная оси струи с началом на оси $O z$, имеющая смысл радиуса струи. Область свечения струи обладает осесимметричной структурой и имеет линейные размеры $\sim 4 \times 6 \mathrm{~mm}$. Поскольку взаимодействие разлетающегося от лазерной плазмы вещества с газом струи может вносить искажения в наблюдаемую форму струи в области, обращенной к плазме, в дальнейшем будет рассматриваться область струи, находящаяся за осью $O z$ по отношению к плазме. На основании рис. 2 определено наблюдаемое пространственное распределение интенсивности свечения струи в координа$\operatorname{Tax}(r, z)$.

Письма в ЖТФ, 2017, том 43, вып. 9 


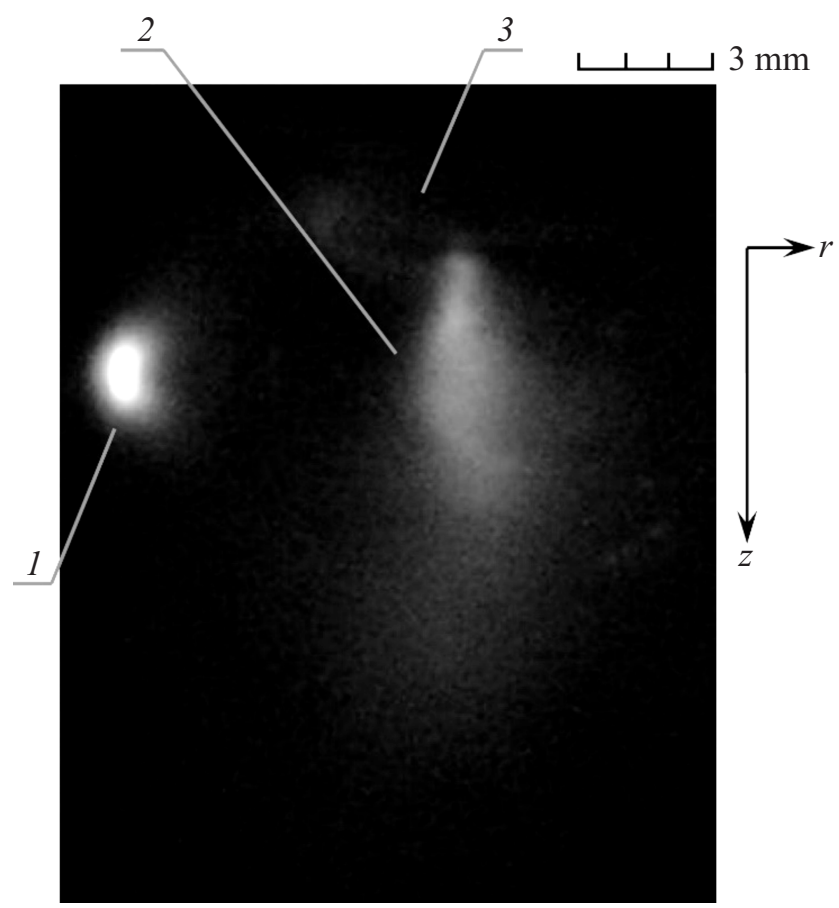

Pис. 2. Полученная в эксперименте фотография свечения струи: 1 - лазерная плазма, 2 - сверхзвуковая струя, 3 - сопло.

Методика определения пространственного распределения концентрации газа внутри струи основана на предположении об осевой симметрии данного распределения. В таком случае наблюдаемая интенсивность свечения струи в каждой точке плоскости $I(r, z)$ является интегралом плотности излучения газа в струе по лучу зрения, направленному к регистрирующему прибору и соответствующему координатам $(r, z)$. Поскольку данные, полученные в эксперименте, не содержат информацию о спектральном составе и абсолютных значениях интенсивности собственного свечения струи, а также параметрах излучения лазерно-плазменного источника, их использование позволяет определить только форму распределения газовой плотности, а все расчеты

Письма в ЖТФ, 2017, том 43, вып. 9 
выполняются в относительных единицах. Выражение для наблюдаемой интенсивности может быть записано в виде

$$
I(r, z)=\int_{r}^{R} \frac{i(s, r, z)}{\sqrt{s^{2}-r^{2}}} s d s,
$$

где $R$ - максимальный радиус струи, $i(s, r, z)$ - плотность интенсивности излучения в точке струи, находящейся на расстоянии $s$ от оси струи и лежащей на луче зрения, который соответствует координатам $(r, z)$. При этом величина $i(s, r, z)$ пропорциональна произведению

$$
i(s, r, z) \sim n(r, z) J(s, r, z),
$$

где $n(r, z)$ - искомая концентрация газа, $J(s, r, z)$ - функция, описывающая плотность потока излучения лазерно-плазменного источника в рассматриваемой точке.

Выражение (1) представляет собой интегральное уравнение Абеля, таким образом, методика нахождения распределения концентрации газа в струе $n(r, z)$ сводится к численному решению уравнения (1) для каждого рассматриваемого значения координаты $z$ с использованием полученных экспериментальных данных о наблюдаемом пространственном распределении интенсивности свечения струи $I(r, z)$. Для численного решения уравнения (1) в данной работе использовался метод конечных элементов на основе обобщенной формулы левых прямоугольников [8], основанный на последовательном определении значения $n(r, z)$ при движении от периферии струи к ее центру.

Результаты расчетов, выполненных на основании полученного экспериментально пространственного распределения интенсивности свечения струи, показаны на рис. 3. На рис. 3, а представлен полученный профиль распределения плотности газа в сверхзвуковой струе. Видно, что в области за соплом уменьшение концентрации газа незначительно на длине порядка 2 mm, что составляет два калибра сопла, а на бо́льших расстояниях профиль расплывается, и концентрация газа падает.

Полученные результаты уместно сравнить с расчетами в работе [6], где выполнен расчет концентрации атомов в струе ксенона путем численного гидродинамического моделирования истечения осесимметричной струи сжимаемого газа из сопла в вакуум. В расчетах [6] параметры генерации струи (давление ксенона в камере высокого

Письма в ЖТФ, 2017, том 43, вып. 9 

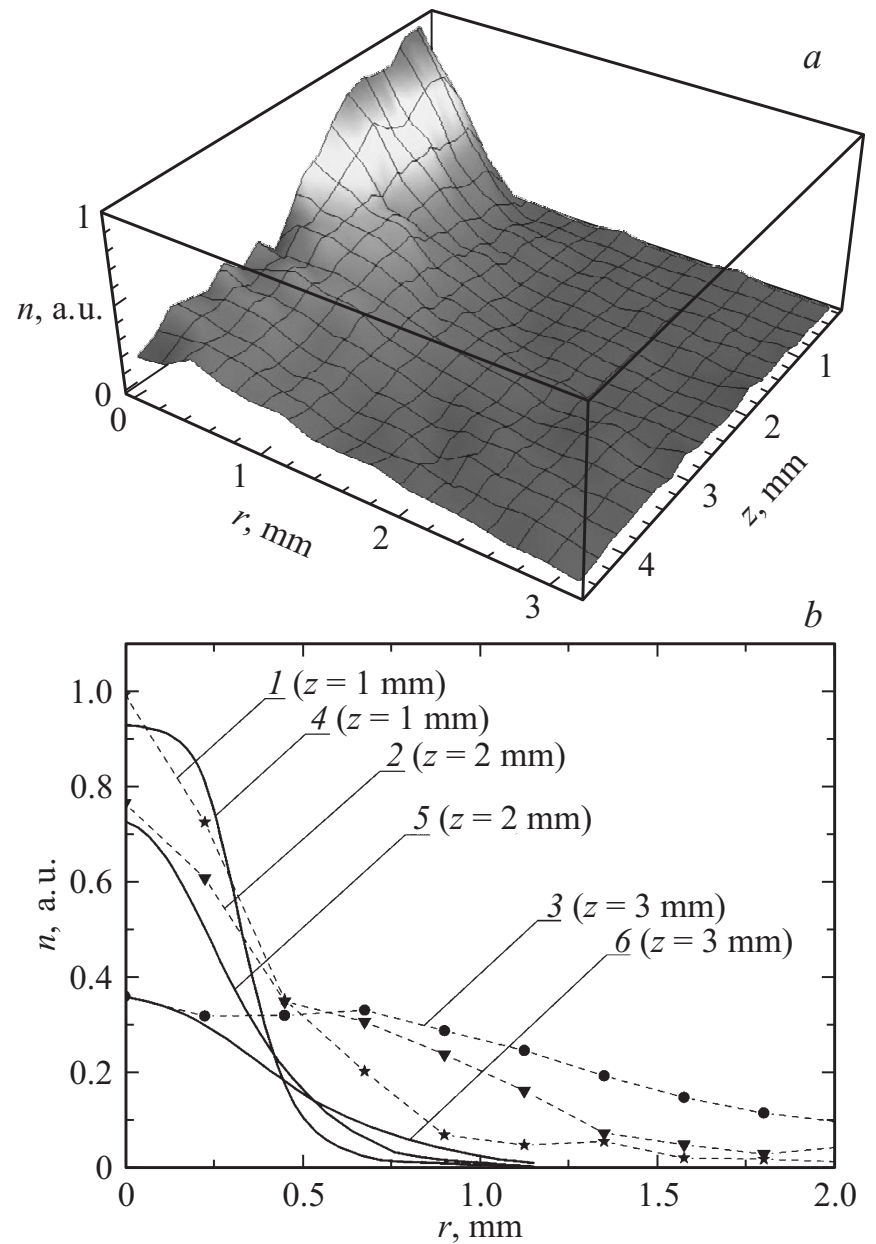

Pис. 3. $a$ : полученное распределение концентрации газа в сверхзвуковой струе. $b$ : $1,2,3$, - радиальные профили концентрации газа в струе, определенные по представленной методике для значений $z=1,2$ и $3 \mathrm{~mm}$ соответственно; 4,5,6 - радиальные профили концентрации газа в струе, полученные в работе [6] для значений $z=1,2$ и $3 \mathrm{~mm}$ соответственно, нормированные на величину $n_{0}=6.05 \cdot 10^{18} \mathrm{~cm}^{-3}$.

Письма в ЖТФ, 2017, том 43, вып. 9 
давления, геометрия сопла Лаваля и другие) практически совпадают с параметрами эксперимента, описанного выше. Сравнение радиальных профилей концентрации, полученных в настоящей работе и в работе [6], представлено на рис. $3, b$. Кривые 1,2 и 3 соответствуют радиальным профилям концентрации газа, определенным по описываемой методике для значений $z=1,2$ и $3 \mathrm{~mm}$. Данные из работы [6] для тех же значений $z$ (кривые 4,5 и 6) приведены в единицах, нормированных на значение концентрации в точке выхода струи из сопла, которое составляет $n_{0}=6.05 \cdot 10^{18} \mathrm{~cm}^{-3}$. В приосевой области струи поведение полученного распределения концентрации ксенона согласуется с данными из работы [6]. В периферийных областях струи полученные значения концентрации значительно превосходят теоретические. Это может быть связано с несовершенством экспериментальной методики визуализации струи. Время свечения ксенона в видимом диапазоне после взаимодействия с излучением лазерной плазмы составляет единицы $\mu \mathrm{s}$ [9], поэтому регистрация интегральной по времени картины свечения струи приводит к расплыванию профиля свечения в результате движения газа. Избыточное свечение периферийных областей струи может быть вызвано газом, поступающим в вакуумную камеру в течение времени открытия газового клапана до момента образования лазерной плазмы.

Сравнивая результаты, полученные в настоящей работе, с расчетными данными [6], следует отметить, что предложенная методика определения концентрации атомов в струе правильно отражает основную особенность адиабатического расширения газа при выходе из сверхзвукового сопла. Тепловая энергия газа переходит в энергию направленного движения, при этом формируется плотная и холодная центральная часть (керн) и периферия из теплого газа, плотность которого быстро спадает.

Таким образом, предложенная в работе методика позволяет качественно определять распределение концентрации атомов в струе при истечении в вакуум и сравнивать режимы работы различных устройств, генерирующих струю. Ее использование дает, в частности, возможность выбирать оптимальный режим истечения для использования струи в качестве мишени в лазерно-плазменных источниках коротковолнового излучения.

Дальнейшее усовершенствование методик визуализации и формирования струи (использование короткой выдержки (менее $1 \mu \mathrm{s}$ ) и быстродействующего газового клапана) позволит повысить качество

Письма в ЖТФ, 2017, том 43, вып. 9 
определения распределения концентрации газа в струе. В данной работе использовался ксенон, однако следует отметить, что, поскольку излучение лазерной плазмы обладает широким спектром и высокой интенсивностью, то предложенная методика применима к сверхзвуковым струям других атомарных и молекулярных газов.

Авторы М.В. Петренко и С.В. Бобашев благодарят С.Г. Калмыкова за полезные обсуждения.

\section{Список литературы}

[1] Капралов В.Г., Корде Р., Левашов В.Е. и др. // Квант. электрон. 2002. Т. 32. B. 2. C. 149.

[2] de Bruijn R., Koshelev K., Bijkerk F. // J. Phys. D: Appl. 2003. V. 36. P. L88.

[3] Домрачева И.В., Петренко М.В., Степанова З.А. и др. // Письма в ЖТФ. 2007. T. 33. В. 22. С. 9.

[4] Забродский В.В., Задиранов Ю.М., Калмыков С.Г. и др. // Письма в ЖТФ. 2014. T. 40. B. 15. C. 38.

[5] Wachulak P.W., Bartnik A., Fiedorowicz H. // Opt. Lett. 2010. V. 35. N 14. P. 2337.

[6] Гарбарук А.В., Демидов Д.А., Калмыков С.Г., Сасин М.Э. // ЖТФ. 2011. Т. 81. B. 6. C. 20.

[7] Richardson M. // Vacuum ultraviolet spectroscopy I. Academic Press, San-Diego, CA, USA, 1998. P. 83-92.

[8] Сизиков В.С., Смирнов А.В., Федоров Б.А. // Изв. вузов. Математика. 2004. N 8. C. 62.

[9] Калмыков С.Г., Мирошников И.В., Петренко М.В., Сасин М.Э. // Письма в ЖТФ. 2011. Т. 37. В. 6. С. 63. 